\title{
Phosphodiesterases in the rat ovary: effect of cAMP in primordial follicles
}

\author{
Tonny Studsgaard Petersen ${ }^{1,2}$, Martin Stahlhut ${ }^{2}$ and Claus Yding Andersen ${ }^{1}$ \\ ${ }^{1}$ Laboratory of Reproductive Biology, The Juliane Marie Centre for Women, Children, and Reproduction, \\ Copenhagen University Hospital, Copenhagen University, Department 5712, Blegdamsvej 9, \\ Copenhagen 2100, Denmark and ${ }^{2}$ LEO Pharma, Ballerup 2750, Denmark
}

Correspondence should be addressed to T S Petersen; Email: tsp@person.dk

\begin{abstract}
Phosphodiesterases (PDEs) are important regulators of the intracellular cAMP concentration, which is a central second messenger that affects a multitude of intracellular functions. In the ovaries, cAMP exerts diverse functions, including regulation of ovulation and it has been suggested that augmented cAMP levels stimulate primordial follicle growth. The present study examined the gene expression, enzyme activity and immunolocalization of the different cAMP hydrolysing PDEs families in the rat ovary. Further, the effect of PDE4 inhibition on primordial follicle activation in cultured neonatal rat ovaries was also evaluated. We found varied expression of all eight families in the ovary with Pde7b and Pde8a having the highest expression each accounting for more than $20 \%$ of the total PDE mRNA. PDE4 accounted for 15-26\% of the total PDE activity. Immunoreactive PDE11A was found in the oocytes and PDE2A in the corpora lutea. Incubating neonatal rat ovaries with PDE4 inhibitors did not increase primordial follicle activation or change the expression of the developing follicle markers Gdf9, Amh, Inha, the proliferation marker Mki67 or the primordial follicle marker Tmeff2. In addition, the cAMP analogue 8-bromo-cAMP did not increase AKT1 or FOXO3A phosphorylation associated with follicle activation or increase the expression of Kitlg known to be associated with follicle differentiation but did increase the Tmeff2, Mki67 and Inha expression in a dose-dependent manner. In conclusion, this study shows that both Pde7b and Pde8a are highly expressed in the rodent ovary and that PDE4 inhibition does not cause an increase in primordial follicle activation.
\end{abstract}

Reproduction (2015) 150 11-20

\section{Introduction}

cAMP is a fundamental intracellular second messenger involved in signal transduction for many hormones, growth factors and neurotransmitters (Zaccolo 2011). In the ovaries, cAMP is of utmost importance being instrumental as a second messenger for the follicle stimulating hormone $(\mathrm{FSH})$ and luteinising hormone $(\mathrm{LH})$ receptors, and is central in growth and development of follicles in all stages (Conti 2002, Zhang et al. 2004, Menon \& Menon 2012).

Inactivation of CAMP is carried out by phosphodiesterases (PDEs), which exists as 11 families of enzymes each with one to four members. Each family differs in regulation and specificity for CAMP and cGMP. PDE1-4, PDE7-8 and PDE10-11 all catalyse hydrolysis of cAMP, while the remaining three PDE families solely hydrolyse cGMP (Azevedo et al. 2014). In the rodent ovary, PDE4D and PDE3 are known to exert important roles in regulation of oocyte maturation and ovulation (Tsafriri et al. 1996, Park et al. 2003, Conti et al. 2012). In addition, the presence of PDE8 has been reported in the bovine and human ovary (Sasseville et al. 2009, Petersen et al. 2015) but has not been studied in rodents.

Elevated levels of cAMP have also been shown to promote folliculogenesis and follicle survival in cultured human ovarian cortex stimulated with the cAMP analogue 8-bromo-cAMP (Zhang et al. 2004). The exact mechanism responsible for primordial follicle activation to enter growth is uncertain but recent studies have shown that the PI3Kinase/AKT1/mTORC1/ FOXO3A pathway is involved (Oktem \& Urman 2010). Activation of this pathway leads to phosphorylation of both AKT1 (RAC-alpha serine/threonine-protein kinase) and its downstream target FOXO3A (Forkhead box protein O3). The latter is a transcription factor that upon phosphorylation is exported from the nucleus allowing transcription and follicle activation to take place. This occurs within $6 \mathrm{~h}$ after activation of the pathway (Li et al. 2010). It is possible that cAMP interacts with the PI3Kinase pathway through exchange protein directly activated by CAMP (EPAC) in the oocyte as seen in skeletal muscle (Baviera et al. 2010). Alternatively, cAMP may increase the production of Kit ligand (KITLG), 
also known as stem cell factor, in the granulosa cells (GC) as gene expression of KITLG has been shown to be stimulated by cAMP in Sertolli cells, and in ovarian cancer cells (Shaw et al. 2002, Grimaldi et al. 2003). KITLG can also activate AKT though its receptor cKIT located on the oocyte (De Miguel et al. 2002).

In addition to the effects in the reproductive system, PDEs exert functions in many other cells located in a number of organs. PDE inhibitors have attracted a great deal of attention as possible drug candidates for treatment of many diseases such as inflammatory and neurological diseases. However, the majority of PDEs inhibitors have failed to reach the market due to adverse effects (Gavalda \& Roberts 2013, Azevedo et al. 2014). Inhibition of PDE4 is known to affect ovulation and the activity of corpora lutea $(\mathrm{CL})$ in rodents (Park et al. 2003, McKenna et al. 2005, Losco et al. 2010) but a possible stimulatory effect on the activation of primordial follicle growth mediated by cAMP would be regarded as a serious side effect due to its irreversible nature. The effects on the ovary by PDE4 inhibitors are most likely caused by elevated cAMP, but the presence of other CAMP hydrolysing PDEs in the follicle may alter the effect of specific PDE4 inhibitors and limited information is available about other members of PDEs in the rodent ovary.

The aim of the present study was to provide basic information on the presence, expression and immunolocalization of all cAMP hydrolysing PDEs during folliculogenesis in rats and to study the effect of PDE4 inhibition on primordial follicle activation in an in vitro neonatal rat model.

\section{Materials and Methods Animals}

Ovaries were isolated from day 4 post-natal (PN) Han-wistar rats (Harlan, Horst, The Netherlands) as previously published (Petersen \& Andersen 2014). For isolation of GC, 10-week-old Wistar rats (Taconic, Ejby, Denmark) were stimulated with 22.5 IU FSH/LH (Menopur, Ferring, Copenhagen, Denmark) and two groups were injected after $46 \mathrm{~h}$ with $20 \mathrm{IU}$ human chorionic gonadotropin (hCG) (Pregnyl, MSD, Ballerup, Denmark). After $2 \mathrm{~h}$ or $4 \mathrm{~h}$, the animals were sacrificed and the ovaries removed. The third group acting as control did not receive hCG and was sacrificed after $48 \mathrm{~h}$. Individual follicles were punctured and the GC were isolated with a Pasteur pipette and washed repeatedly to remove blood contamination. The cells were snap-frozen and stored at $-80^{\circ} \mathrm{C}$ for later RNA isolation or PDE activity measurement.

Follicles from Han-Wistar rats PN day 8-10 and CL from mature rats were isolated enzymatically by incubating the ovaries in MEM alpha media (Life Technologies) supplemented with $0.1 \% \mathrm{w} / \mathrm{v}$ Collagenase (Sigma-Aldrich) and $0.8 \% \mathrm{w} / \mathrm{v}$ DNase (Sigma-Aldrich) for $20 \mathrm{~min}$ at $37^{\circ} \mathrm{C}$. The ovaries were transferred to PBS containing $5 \% \mathrm{v} / \mathrm{v}$ foetal bovine serum (FBS) (Life Technologies), incubated for $10 \mathrm{~min}$ and transferred to fresh culture media where the follicles and $\mathrm{CL}$ were dissected free with a pair of $30 \mathrm{G}$ hypodermic needles. Sequential filtering of the media though a 40 and $11 \mu \mathrm{m}$ nylon filter (Millipore, Hellerup, Denmark) was used to separate follicles into two groups of 11-40 and 40-300 $\mu \mathrm{m}$ in diameter, a method previously used with primate ovaries (Hornick et al. 2012).

The Danish Animal Experiments Inspectorate (Dyreforsøgstilsynet) approved the study.

\section{Culture}

The ovaries were cultured in Nunc four well dishes (Fisher Scientific, Hvidovre, Denmark) on $12 \mathrm{~mm}$ floating hydrophilic PTFE cell culture insert with pore size of $0.4 \mu \mathrm{m}$ (Millipore) covered with a small drop of media. The culture media consisted of MEM alpha, $1 \% \mathrm{v} / \mathrm{v}$ human serum albumin (CSL Behring, Lyngby, Denmark), $1 \% \mathrm{v} / \mathrm{v}$ insulin-transferrinselenium (Life Technologies), 1\% v/v Pen Strep (Life Technologies), $1 \% \mathrm{v} / \mathrm{v}$ glutamax (Life Technologies) and 1\% v/v FBS, 25 IU/I FSH (Puregon, MSD, Ballerup, Denmark). Each well contained $200 \mu \mathrm{l}$ medium and the media was changed every other day. As selective PDE4 inhibitors, the two substances LEO29102 and its pyridine N-oxide LEO28386 (LEO Pharma, Ballerup, Denmark) were used (Felding et al. 2014). The inhibitors were dissolved in DMSO (Sigma-Aldrich) and diluted in culture media until the final DMSO concentration was $0.01 \%$. For experiments with the CAMP analogue 8-bromocAMP (Santa Cruz Biotechnology, Santa Cruz, CA, USA), DMSO was replaced with water. The ovaries were removed after culturing for $6 \mathrm{~h}$ for immunohistochemistry, $2 \mathrm{~h}$ for western blotting and 7 days for qPCR and follicle classification. Briefly, the ovaries were washed in PBS and snap-frozen in liquid nitrogen or embedded in $2 \%$ agarose and fixated in Bouin's solution.

\section{RNA extraction and RT-qPCR}

RNA extractions were performed with the Dynabeads mRNA DIRECT kit (Life Technologies) according to the manufactures instructions with the addition of $1 \% \mathrm{v} / \mathrm{v}$ protease inhibitor cocktail for use with mammalian cell and tissue extracts (Sigma-Aldrich) to the lysis buffer. $200 \mu$ l lysis buffer and washing buffer were used and the mRNA was eluted in $25 \mu$ l elution buffer. RT was carried out using the High capacity cDNA kit (Life Technologies). Real-time quantitative PCR was performed as described previously (Petersen \& Andersen 2014) with TaqMan primer probe assays (Assay ID) against Pde1b (Rn00575591_m1), Pde1c (Rn00579334_m1), Pde2a (Rn01648917_m1), Pde3a (Rn00569192_m1), Pde3b (Rn00568191_m1), Pde4a (Rn00565354_m1), Pde4b (Rn00 566785_m1), Pde4c (Rn01754406_m1), Pde4d (Rn00566 798_m1), Pde7a (Rn01493469_m1), Pde7b (Rn00590117_ m1), Pde8a (Rn01648408_m1), Pde8b (Rn01489992_m1), Pde10a (Rn00673152_m1), Pde11a (Rn01639097_m1), Gdf9 (Rn00572328_m1), Kitlg (Rn01502851_m1), Inha (Rn00 561423_m1), Tmeff2 (Rn02110772_s1), Amh (Rn005 63731_g1), Rpl32 (Rn00820748_g1), Mki67 (Rn01451 446_m1) and Gapdh (Rn01775763_g1). The expression of 
Pde1b-Pde11a was normalised to the total amount of PDE mRNA according to the following equation:

$$
\begin{aligned}
\operatorname{Proportion}_{\mathrm{Pde}_{i}}= & \frac{2^{-C q_{\mathrm{Pde}_{i}}}}{\sum_{j} 2^{-C q_{\mathrm{Pde}_{j}}}}, i, j \\
& \in\{1 b, 1 c, 2 a, \ldots, 8 b, 10 a, 11 a\} .
\end{aligned}
$$

The amplification efficiency for the TaqMan assays according to the manufacture is $100 \%$ (http://docs.appliedbiosystems. com/pebiodocs/00113186.pdf). This was validated by linear regression with Gapdh as the reference gene (Cui et al. 2015) with adjustment for sample group (included as an additional independent variable). The expression of the remaining genes is presented as $2^{-\Delta C q}$ normalized to Rpl32 (Schmittgen \& Livak 2008).

\section{PDE activity assay}

GC were lysed in $50 \mathrm{mM}$ Tris- $\mathrm{HCl}(\mathrm{pH} 7.5)$ containing $0.05 \%$ Triton-X 100, $1.7 \mathrm{mM}$ EGTA, $8.3 \mathrm{mM} \mathrm{MgCl} 2,6 \mathrm{mM} \mathrm{CaCl}_{2}$ and $1 \%$ of protease inhibitor cocktail for mammalian cells (SigmaAldrich). Aliquots of the lysate were assayed for total PDE activity as well as roflumilast insensitive (total non-PDE4) activity in the absence or presence of $0.5 \mu \mathrm{m}$ roflumilast (Santa Cruz Biotechnologies). The roflumilast sensitive PDE4 activity was determined by subtracting the roflumilast-insensitive activity from the total activity. The PDE assays were performed using a cAMP-PDE SPA assay as detailed previously (Petersen \& Andersen 2014) with the following modifications: $0.1 \%$ BSA (Sigma-Aldrich) was used in the reaction buffer and the activity was measured once after $15 \mathrm{~min}$.

\section{Western blot}

Ovaries and rat tongue were homogenized using cryogrinding and lysed in RIPA buffer with $1 \%$ v/v protease inhibitor cocktail and $1 \% \mathrm{v} / \mathrm{v}$ phosphatase inhibitors cocktail 3 (Sigma-Aldrich). Protein concentration was determined using the Bio-Rad DC Protein assay (Bio-Rad). Total protein of $40 \mu \mathrm{g}$ for PN day 8 ovaries and tongue, and $13 \mu \mathrm{g}$ for PN day 6 ovaries were loaded on a Bis-Tris $4-12 \%$ Gel and electrophoretic separated. The proteins were transferred to an Invitrolon PVDF membrane (Life Technologies) and equal protein load was checked using CPTS stain (Bickar \& Reid 1992) followed by destaining in a $5 \% \mathrm{w} / \mathrm{v}$ BSA (Sigma-Aldrich) solution in Tris Buffed Saline and drying of the membrane after an ethanol wash. Western blotting was carried out as described previously (Naryzhny 2009) using rabbit antibodies raised against FOXO3A (AbCam, Cambridge, UK), phospho-FOXO3A (AbCam), phosphoAKT1 S473 (AbCam) and peroxidase labelled donkey anti-rabbit secondary antibody (Agrisera, Vännas, Sweden). The blot was developed with SuperSignal West Femto Substrate (Thermo Fisher Scientific, Hvidovre, Denmark) and imaged with a Canon EOS $1100 D$ camera (Canon, Tokyo, Japan) cooled with dry ice.

\section{Staining and follicle classification}

The fixated ovaries were dehydrated and embedded in paraffin, cut in $10 \mu \mathrm{m}$ serial sections and stained with haematoxylin and PAS. The slides were digitalized using a Pannoramic MIDI slide scanner (3DHISTEC, Budapest, Hungary) equipped with a Plan-Apochromat $20 \times / 0.8$ objective (Carl Zeiss, Jena, Germany) and the sections were isolated and aligned using custom software. The follicles were classified based on the

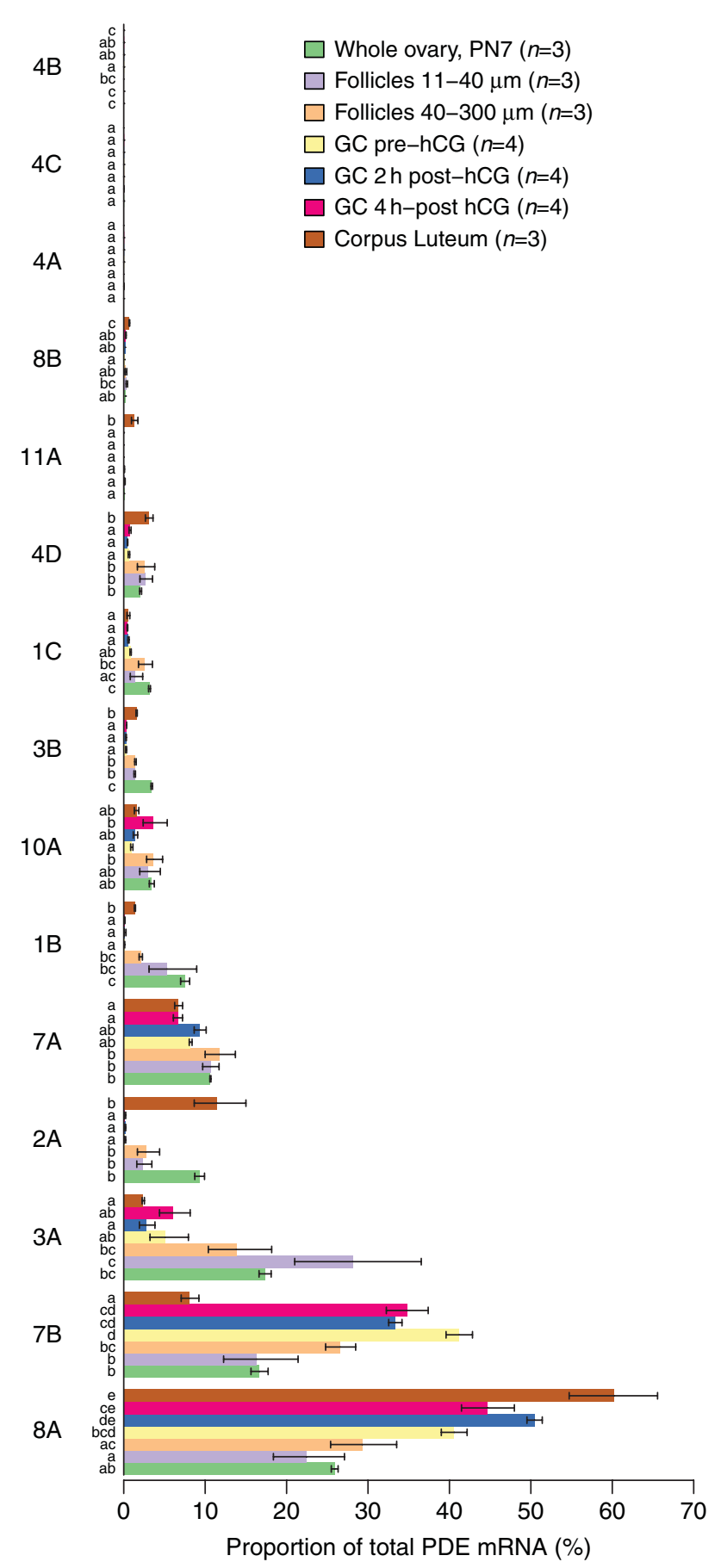

Figure 1 Gene expression of cAMP hydrolysing PDEs in the rat ovary evaluated by RT qPCR. Follicles were isolated from rats PN day 8-11. Corpora lutea from mature rats and granulosa cells from $\mathrm{FSH}+\mathrm{LH}$ stimulated adult rats at various timepoints after hCG stimulation. The expression was normalized to the total PDE mRNA amount. Different letters indicate statistical significant difference. Error bars indicate S.E.M. 
Table 1 Total and type 4 cAMP phosphodiesterase activity in rat granulosa cells before and after hCG stimulation.

\begin{tabular}{lcc}
\hline & $\begin{array}{c}\text { Total PDE activity } \\
\text { (pmol/min per } \mathrm{mg} \\
\text { total protein) }\end{array}$ & $\begin{array}{c}\text { PDE4 activity } \\
\text { (pmol/min per mg } \\
\text { total protein) }\end{array}$ \\
\hline Pre-hCG & $42.0 \pm 7.2$ & $6.5 \pm 1.9$ \\
2 h post-hCG & $39.7 \pm 2.5$ & $9.1 \pm 3.7$ \\
4 h post-hCG & $37.6 \pm 2.9$ & $9.9 \pm 2.2$ \\
\hline
\end{tabular}

Mean \pm S.E.M., $n=5$.

appearance of the follicles according to Lintern-Moore et al. (1974) into five categories: primordial with flatten GC (class B), intermediary with at least one cuboid GC (class B/C), primary with all GC being cuboidal (class C), secondary with more than one layer of GC (class D1) and antral with visible antrum (class D2-F). Follicle classification and area measurement were performed on oocytes with visible nucleoli on every fifth section using TrakEM2 (Cardona et al. 2012).

\section{Immunohistochemistry analysis}

For IHC detection of PDEs, sections were deparaffinised with xylene and rehydrated though ethanol series. Antigen retrieval was performed in citrate buffer, $\mathrm{pH} 6$ (Dako, Glostrup, Denmark) for PDE3A and PDE4D. The slides were blocked in an antibody dilution buffer (Dako) followed by incubation of primary antibodies diluted in antibody blocking buffer overnight at $4{ }^{\circ} \mathrm{C}$. The slides were washed and detected using Dako envision and $\mathrm{DAB}+$. Haematoxylin was used for nuclear counter stain. The primary antibodies raised in rabbits against PDE1B, PDE1C, PDE2A, PDE3A, PDE3B, PDE4A, PDE4B, PDE4C, PDE4D, PDE7A, PDE7B, PDE8A, PDE8B and PDE11A were all purchased from FabGennix (Frisco, TX, USA). The images were captured using a Pannoramic MIDI Slide scanner. For detection of FOXO3A, no blocking was used, antigen retrieval was performed with Tris-EDTA pH 9 and the washing buffer used was water with $0.5 \%$ v/v Tween-20 (Merck). AntiFOXO3A (AbCam) was used as the primary antibody and peroxidase labeled donkey anti-rabbit as secondary antibody. $\mathrm{DAB}+($ Dako) was used for detection. Images were captured with a CANON EOS $1100 \mathrm{D}$ camera mounted on an Olympus IX-70 microscope (Olympus) equipped with an Olympus UAPO N $34040 \times / 1.15$ Water immersion objective.

\section{Statistical analysis}

ANOVA was used for comparing differences in gene expression with adjustment for multiple comparisons using the Benjamini-Hochberg correction and with a logit transformation applied to the relative PDE mRNA gene expression (Warton \& Hui 2011). $\chi^{2}$ was used for comparing differences in follicle class distribution. All values are expressed as mean \pm s.E.m. A $P$ value $<0.05$ was considered to be statistical significant.

\section{Results \\ qPCR and PDE activity}

In the neonatal ovary, Pde8a was the highest expressed PDE, followed by Pde3a, Pde7b and Pde7a (Fig. 1). The remaining PDEs each accounted for $<10 \%$ of the total PDE mRNA. Pde4a, Pde4b, Pde4c, Pde8b and Pde11a had a very low expression in total contribution, $<1 \%$. The smaller follicles $11-40 \mu \mathrm{m}$ diameters followed the same pattern although Pde3a was the highest expressed PDE followed by Pde8a. In the larger follicles, Pde8a had the highest expression followed by Pde7b, Pde3a and Pde7a, although there were no statistical significant differences between the two follicle groups.

In the GCs, only Pde8a and Pde7b each accounted for more than $10 \%$ of the total PDE mRNA. Only Pde10a was affected by hCG increasing 4.4-fold after $4 \mathrm{~h}$ posthCG. In contrast to the whole ovary and follicles, the expressions of both Pde1b, Pde2a, and Pde $3 b$ were below $1 \%$. The $\mathrm{CL}$ had the highest expression of Pde8a accounting for $60 \%$ followed by Pde2a at $12 \%$. The remaining PDEs each accounted for $<10 \%$ of the total PDE mRNA.

Table 2 Immunohistochemical staining for phosphodiesterases in different types of follicles and in the corpus luteum of the rat ovary.

\begin{tabular}{|c|c|c|c|c|c|}
\hline Antibody raised against & Primordial & Primary & Secondary & Antral & Corpus luteum \\
\hline PDE1B & + & + & + & - & ++ \\
\hline PDE1C & + & + & + & + & ++ \\
\hline PDE2A & - & - & - & - & + \\
\hline PDE3A & $++^{a}$ & $++^{\mathrm{a}}$ & $++^{\mathrm{a}}$ & + & + \\
\hline PDE3B & $++^{a}$ & $++^{a}$ & $++^{a}$ & $++^{b}$ & + \\
\hline PDE4A & - & - & $+^{a}$ & $+^{a}$ & ++ \\
\hline PDE4B & + & + & + & + & + \\
\hline PDE4C & $++^{a}$ & $++^{a}$ & $++^{a}$ & $++^{a}$ & + \\
\hline PDE4D & + & + & + & + & + \\
\hline PDE7A & $+^{a}$ & $+^{a}$ & $+^{a}$ & $+^{a}$ & + \\
\hline PDE7B & $+^{a}$ & $+^{a}$ & $+^{a}$ & $+^{a}$ & + \\
\hline PDE8A & ++ & ++ & ++ & ++ & + \\
\hline PDE8B & - & - & - & $+++^{b}$ & + \\
\hline PDE11A & $+++^{\mathrm{a}}$ & $+++^{a}$ & $++^{a}$ & $++^{\mathrm{a}}$ & + \\
\hline
\end{tabular}

Staining intensity: +++ , strong staining; ++ , moderate staining; + , weak staining; - , no staining.

${ }^{\mathrm{a}}$ Oocyte localization. ${ }^{\mathrm{b}}$ Theca cells. 


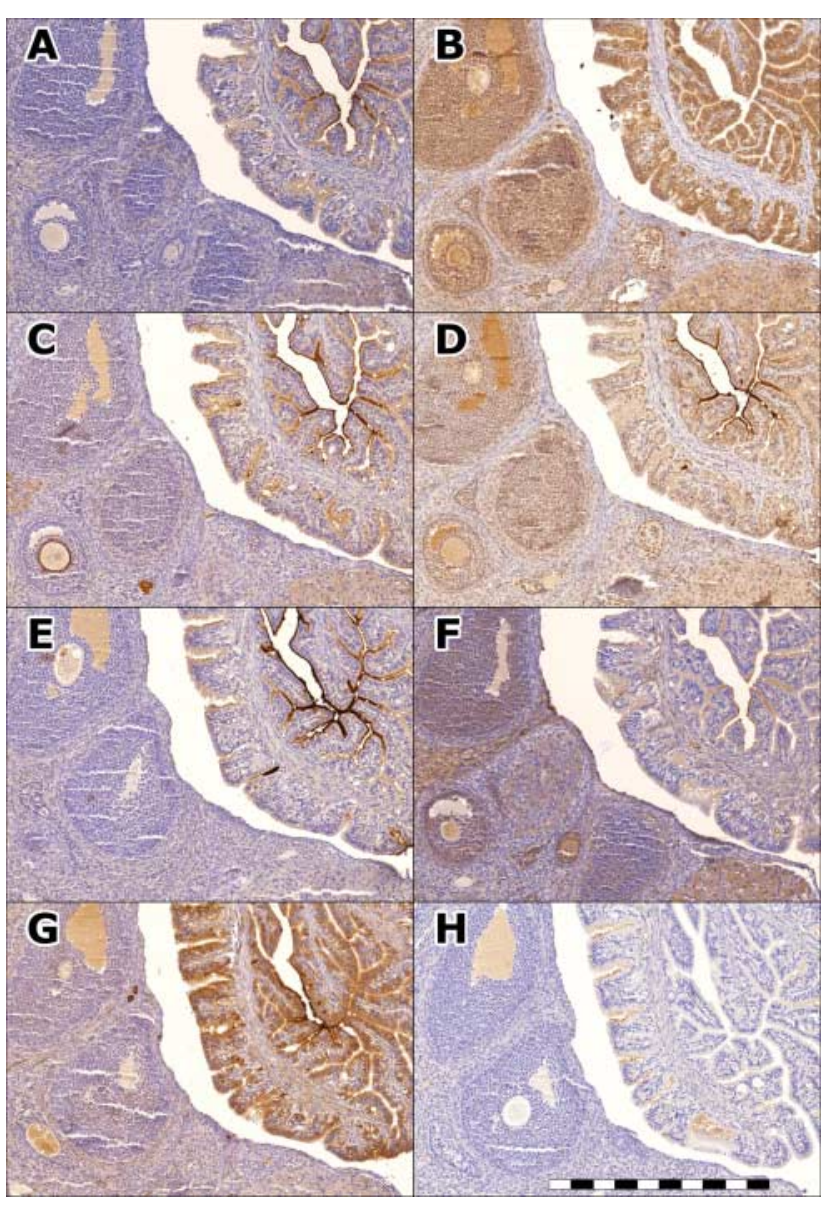

Figure 2 Immunohistological staining for selected PDEs in adult rat ovary. PDE2A was present in the corpora lutea (A), PDE3A in follicles and corpora lutea (B), PDE3B showed string staining in the oolemma (C), PDE4D was present in follicles and corpora lutea (D), PDE7B stained faintly in the corpora lutea and follicles (E), PDE8A stained both follicles and corpora lutea (F), PDE11A stained primordial follicles strongly $(\mathrm{G})$, the control without primary antibody stained the follicle fluid faintly $(\mathrm{H})$. Scale bar $1 \mathrm{~mm}$.

In all groups, the expression of Pde4a, Pde $4 b$ and $P d e 4 c$ were very low. Pde $4 d$ accounted for $0.5-3.2 \%$.

In contrast, the enzymatic activity of PDE4 accounted for $15-26 \%$ of the total PDE activity in the GCs with a tendency to a higher activity post-hCG (Table 1 ).

\section{IHC}

The staining patterns for various PDEs are summarized in Table 2. PDE2A was present in the CL (Fig. 2A). PDE3A was detected in the oocytes and with lower intensity in the GC (Fig. 2B). PDE3B stained strongly in the oolemma (Fig. 2C). PDE4D was present in follicles and $\mathrm{CL}$ (Fig. 2D). Positive staining of PDE7B was seen in the oocytes (Fig. 2E), while PDE8A was present in $\mathrm{CL}$ and in follicles (Fig. 2F). Strong staining for PDE11A was observed in oocytes (Fig. 2G).

\section{Follicle activation}

No effect of the two PDE4 inhibitors LEO29102 and LEO28386 was seen on the distribution of follicle classes $\left(\chi^{2}=10.7, \quad P=0.09\right)$ after 1 week of culturing as compared to controls (Fig. 3). The mean follicle diameter was lower in the LEO29102 $(17.9 \pm 0.11 \mu \mathrm{m})$ and LEO28386 $(17.8 \pm 0.11 \mu \mathrm{m})$ groups as compared to control $(18.3 \pm 0.10 \mu \mathrm{m}, P<0.01$ for both groups). In addition, no effect was seen on the expression of Kitlg, Inha, the growing follicle markers Gdf9, Amh, the proliferation marker Mki67 or the primordial follicle marker Tmeff2 (Fig. 4). The cAMP analogue 8-bromocAMP increased Mki67, Inha, Tmeff2 and decreased Amh in a dose-dependent manner, while Gdf9 and Kitlg were unchanged after 1 week of treatment.

In the ovaries cultured for $6 \mathrm{~h}$, no nuclear export of FOXO3A was observed following exposure to $1 \mathrm{mM}$ 8-bromo-cAMP, or $1 \mu \mathrm{M}$ of the PDE4 inhibitor LEO29102 or LEO28386 (Fig. 5). Furthermore, western blot analysis was unable to detect phosphorylation of FOXO3A or AKT1 in ovaries exposed to $100 \mu \mathrm{M}$ 8-bromo-cAMP for $6 \mathrm{~h}$ (Fig. 6).

\section{Discussion}

This study showed that all cAMP hydrolysing PDEs families were expressed in the rat ovary with Pde3a, $P d e 7 a / b$ and Pde8a displaying the highest expression. Pde4d was the major PDE type 4 subtype present in the rat ovary but accounted for a modest $0.5-3.2 \%$ of the total cAMP PDE mRNA. In contrast, the PDE4 enzymatic activity was between 15 and $26 \%$ of the total PDE activity in GC.

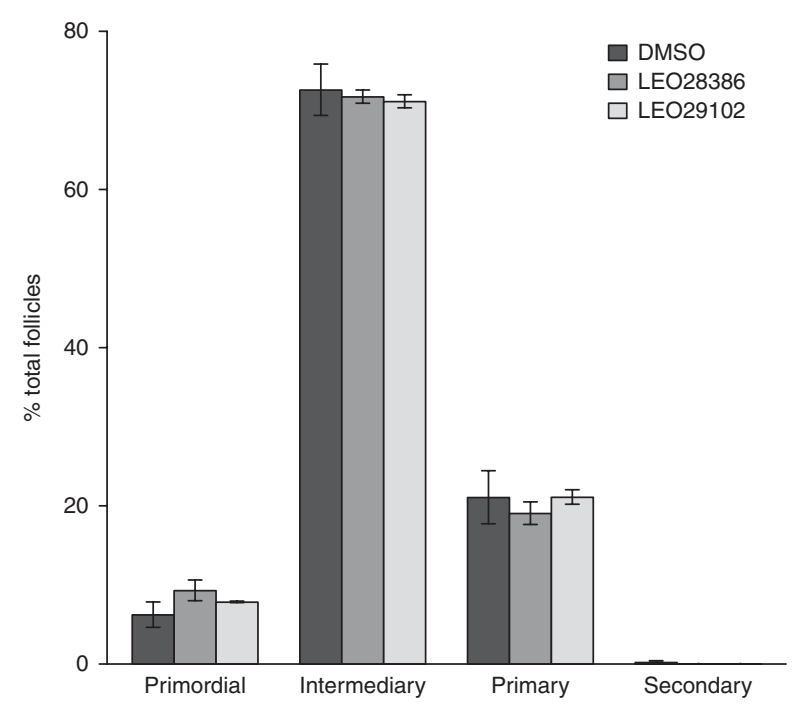

Figure 3 Follicle classification of cultured neonatal rat ovaries. The ovaries were cultured for 7 days in vitro in presence of the PDE4 inhibitor LEO29102 $1 \mu \mathrm{M}$ or LEO28386 $1 \mu \mathrm{M}$, or control (DMSO). Error bars indicates S.E.M. for $n=3$. 

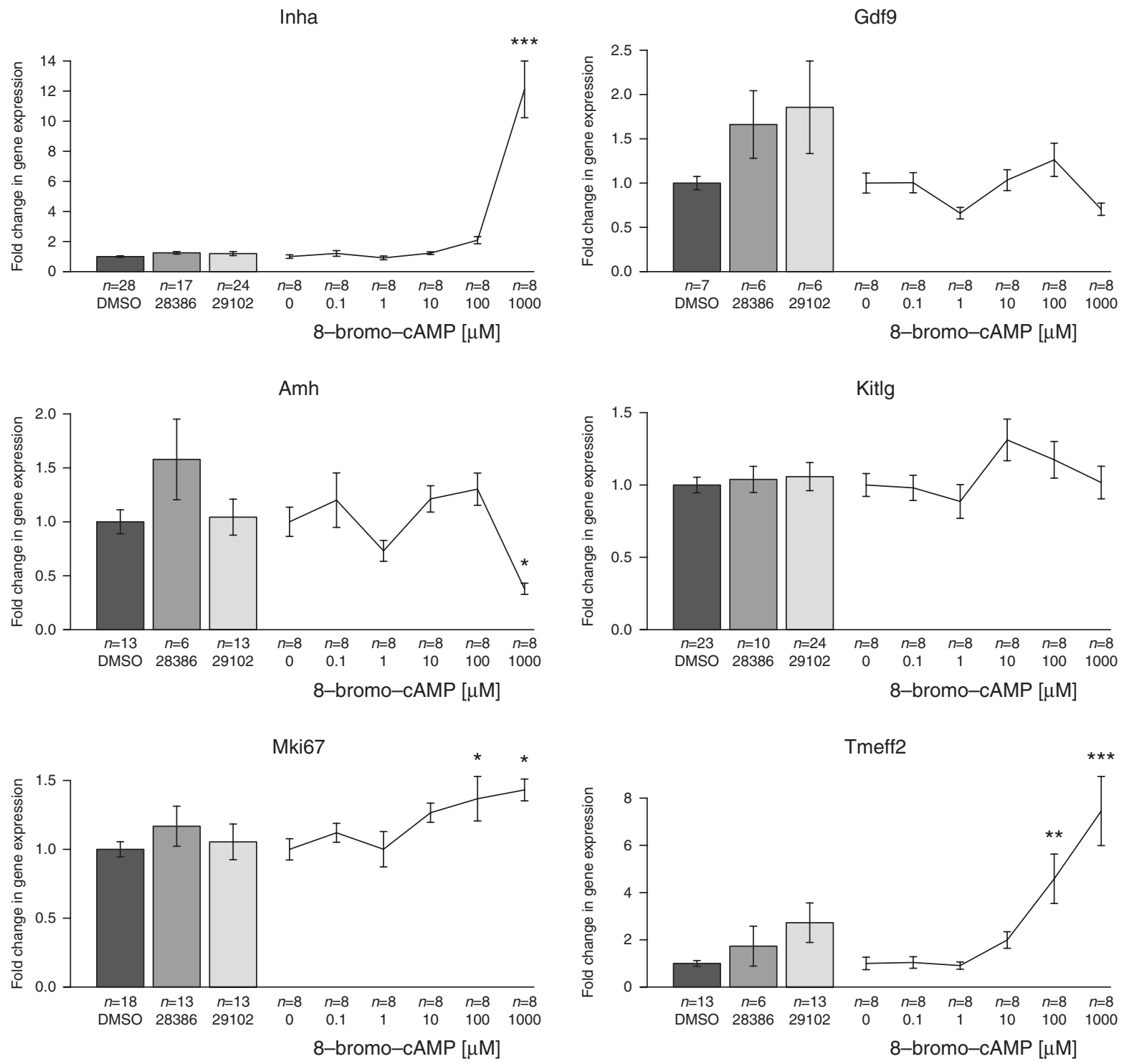

Figure 4 Gene expression of growth and maturation markers in cultured neonatal rat ovaries. The ovaries were cultured for 7 days in vitro in presence of the PDE4 inhibitor LEO29102 $1 \mu \mathrm{M}$ or LEO28386 $1 \mu \mathrm{M}$, or control (DMSO). In addition, the effect of various concentration of the cAMP analogue 8-bromo-cAMP was tested. The following genes were measured: inhibin A, alpha subunit (Inha), growth differentiation factor-9 (Gdf9), antiMüllerian hormone (Amh), Kit ligand (Kitlg), antigen KI-67 (Mki67) and transmembrane protein with EGF-like and two follistatin-like domains 2 (Tmeff2). The gene expression was standardized to the house keeping gene Rpl32 and normalized to the control group. Error bars indicate S.E.M. ${ }^{*} P<0.05 ; * * P<0.01 ; * * * P<0.001$ vs control.

The presence of a number of different PDE families in the ovary and in the follicles allow cells to regulate cAMP tightly at various concentrations due to differences in and post-translational modifications and affinity for CAMP. Both PDE7 and PDE8 are most active at low cAMP concentrations $\left(K_{\mathrm{m}}<100 \mathrm{nM}\right)$ and PDE8 has been suggested to regulate the resting levels of CAMP (Bender \& Beavo 2006, Tsai et al. 2011, Shimizu-Albergine et al. 2012) and it can speculated that PDE7 and PDE8 share the same function in the ovary. In contrast, PDE4 has a lower affinity for cAMP $\left(K_{\mathrm{m}}=1-10 \mu \mathrm{M}\right)$ meaning that this enzyme is mainly active at higher CAMP concentrations observed during hormonal stimulation of cAMP production as seen for instance with hCG stimulation (Park et al. 2003, Bender \& Beavo 2006). This may explain why we were unable to observe a stimulatory effect on primordial follicle activation when inhibiting PDE4. Perhaps the cAMP concentration was too low for optimal PDE4 activity.

Both PDE3 and PDE2 hydrolyse cAMP but are both affected by cGMP although they differ in their effect since PDE2 is stimulated while PDE3 is inhibited by cGMP (Azevedo et al. 2014). The latter is important in the oocyte of the preovulatory follicle, where PDE3 activity 


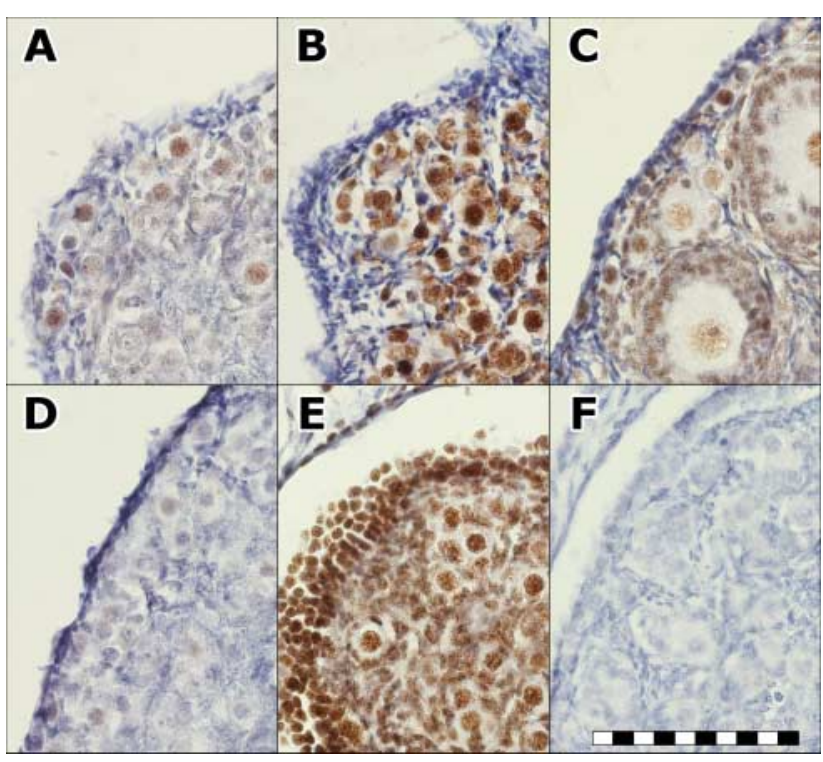

Figure 5 Immunolocalization of FOXO3A after treatment with 8bromo-cAMP or PDE4 inhibitors. Neonatal ovaries were treated with 1 mM 8-bromo-cAMP (B), $1 \mu$ M LEO28386 (C), 1 MM LEO29102 (D), $\mathrm{DMSO}(\mathrm{A})$ for $6 \mathrm{~h}$ or no treatment $(\mathrm{F})$ followed by staining for FOXO3A. Staining with no primary antibody was used as control (F). No nuclear export of FOXO3A was seen in any of the groups. Scale bar $=100 \mu \mathrm{m}$.

is inhibited by cGMP originating from the cumulus cells. After the LH peak, cGMP declines and the PDE3 activity increases leading to a reduction in the cAMP concentration in the oocyte and resumption of meiosis (Vaccari et al. 2009). Although the function of cGMP in the $\mathrm{CL}$ is unclear (Budnik et al. 1987, Johnson et al. 1999), the presence of PDE2 suggests that CGMP may diminish the effect of cAMP by increasing its degradation.

We saw a relative low expression of $P d e 4 d$ compared to the enzymatic activity of PDE4. Recently, similar results have been reported in human GCs (Petersen et al. 2015). This could be due to a rapid turnover of the mRNA as seen with other transcripts involved in signalling (Schwanhausser et al. 2011). This would be advantageous as it allows a fast response to changes in cAMP concentration, e.g. as seen in conjunction with hCG stimulation (Park et al. 2003). Despite not accounting for the majority of the PDE activity in the GC, PDE4 is well known to exert important functions in the rodent ovary (Park et al. 2003, McKenna et al. 2005, Losco et al. 2010). This may be caused by the previous mentioned differences in CAMP affinity or the subcellular distribution of the PDE families (Conti et al. 2014).

We found high levels of Pde3a in the small follicles. This is in line with previous reported in-situ hybridization of rat ovaries were Pde $3 a$ and to a lesser degree Pde $3 b$ were located in the oocytes (Reinhardt et al. 1995). However, it is unclear what the function is in the preantral follicles as the cumulus cells that are essential in controlling the PDE3 activity in the oocyte of larger follicles are not developed at this time.

Low level of Pde8b was seen in contrast to that observed in bovine ovaries (Sasseville et al. 2009) and in humans (Markholt et al. 2012, Petersen et al. 2015). Instead, Pde8a appeared to be the major subtype present, especially in the CL. A higher level of Pde2a was also seen in the $\mathrm{CL}$ supported by the IHC staining. The IHC staining suggested high levels of PDE11A in the oocyte although the mRNA expression was very low in the small preantral follicles.

We saw no effect of the tested PDE4 inhibitors on the in vitro follicle activation after 7 days of culture, neither in the distribution of the different follicle stage in the histological sections nor in genes differential expressed in the folliculogenesis. This is in contrast to previous reports of such an effect of cAMP analogues in humans (Zhang et al. 2004). Neither the PDE4 inhibitors nor 8-bromo-cAMP affected the expression of Kitlg. This is in line with a previous report that CAMP does not stimulate KITLG in GC (Grimaldi et al. 2003) but in contrast to findings from ovarian cancer cells (Shaw et al. 2002). The lack of effect on Inha expression suggests that PDE4 inhibition alone is not able to generate a sufficient elevation of cAMP to cause a biological effect in the neonatal ovaries as the high dose 8-bromo-cAMP did increase Inha expression.

The tumour suppressor TMEFF2 has recently been shown to be highly expressed in oocytes isolated from human primordial follicles (Markholt et al. 2012). Although traditional markers of the preantral follicle stage like Gdf9 and Amh were not augmented in response to 8-bromo-cAMP stimulation, Tmeff 2 expression was dose dependently increased as a result of exposure to 8-bromo-cAMP stimulation. The precise function of TMEFF2 in the early follicle is unknown, but it has been shown to be down-regulated in cancer cells and to inhibit platelet-derived growth factor (PDGF) AA, that stimulates follicle growth (Sleer \& Taylor 2007, Lin et al. 2011, Brito et al. 2012, Chen \& Ruiz-Echevarria 2013). This suggests that TMEFF2 may play a role in maintaining the primordial follicle in a quiescent state.

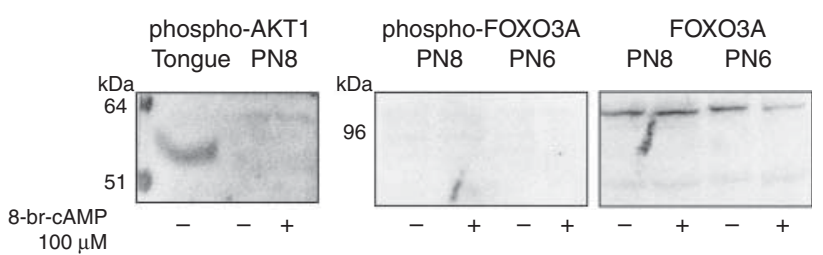

Figure 6 Protein expression of phospho-AKT1 S473, phospho-FOXO3A S253 and FOXO3A after treatment with 8-bromo-CAMP. Western blot of neonatal ovaries cultured for $2 \mathrm{~h}$ in presence of $100 \mu \mathrm{M} 8$-bromoCAMP. Rat tongue was used as positive control for phospho-AKT1. $40 \mu \mathrm{g}$ total protein was used for rat tongue and PN8 ovaries and $13 \mu \mathrm{g}$ total protein for PN6 ovaries. 
In corticotroph cells, TMEFF2 inhibits the cAMP production from CRH (Labeur et al. 2010), suggesting that the increased expression of Tmeff 2 may act as a negative feedback on the increase cAMP level.

We cannot exclude that additional growth factors stimulating CAMP production other than the $25 \mathrm{U} / \mathrm{I} \mathrm{FSH}$ and $1 \%$ FBS may be required in the culture media to obtain a sufficient high cAMP level for PDE4 inhibitors to be effective. Alternatively, inhibition of additional PDEs may be required. Based on the distribution in the small follicles PDE3, PDE7 and PDE8 seem to be obvious candidates.

In the conditions applied in this study, 8-bromo-cAMP did not increase phosphorylation of AKT1, an early marker of follicle activation. The effect of cAMP on the $\mathrm{PI}$ 3Kinase pathway has been described to differ between cell types as the two main effectors of cAMP: protein kinase $A$ and EPAC have been reported to have opposing effects on the pathway (Smith et al. 2005, Chen et al. 2007, Kwak et al. 2008, Baviera et al. 2010). In the present set of experiments, the effect of cAMP seems to be inhibitory on the FOXO3A/AKT1 phosphorylation. An explanation for the previous reported effects of cAMP on primordial follicle activation (Zhang et al. 2004) could be due to changes in hormone/growth factor secretion from later follicular stages affecting the primordial follicles, as the proportion of secondary follicles was higher in the human study. Another important difference between the two studies is the inclusion of $\mathrm{FSH}$ in the culture media in the present study, as additional cAMP may not stimulate the follicle growth further (Ding et al. 2012). However, 8-bromocAMP did increase the expression of Inha suggesting that the ovaries were cAMP responsive. Finally, the culture circumstances in this study might not be optimal for follicular growth although media with similar composition as ours has been used successfully for culturing of rodent ovaries (Wang et al. 2013).

It is well known, that there are species differences in the expression of PDEs in various organs (Bian et al. 2004, Johnson et al. 2012). This poses a problem when translating results obtained in one species to another. The data obtained in this study are likely to prove useful in interpreting data obtained in rat ovaries as information of all PDEs in the ovary has been lacking until now. In addition, the high expression of PDE7 and PDE8 suggests that inhibitors of these families could have an effect on the female reproductive system in rats.

In conclusion, this study showed that in addition to the already known PDE3 and PDE4, the two low $K_{\mathrm{m}}$ PDEs, PDE7 and PDE8 are present in rat ovaries in significant amounts. We did not find any effect of PDE4 inhibition on primordial follicle activation in cultured in vitro neonatal rat ovaries. In addition, 8-bromo-cAMP failed to activate the PI3Kinase/AKT pathway, suggesting that cAMP in itself has no direct effect on initiation of primordial follicles growth.

\section{Declaration of interest}

The authors declare that there is no conflict of interest that could be perceived as prejudicing the impartiality of the research reported.

\section{Funding}

This work was supported by LEO Pharma A/S and The Danish Agency for Science, Technology and Innovation.

\section{Acknowledgements}

The excellent technical help from Christian Møller Olsen, Inga Husum and Sussi Fochhammer is greatly appreciated. I would like to thank my college, Johan Selmer, at LEO Pharma for constructive input, and Kjeld Møllgaard for aiding with the immunohistological staining, Nanna Grand at CiToxLAB for follicle counting. We acknowledge the Core Facility for Integrated Microscopy, Faculty of Health and Medical Sciences, University of Copenhagen.

\section{References}

Azevedo MF, Faucz FR, Bimpaki E, Horvath A, Levy I, de Alexandre RB, Kahn FA, Manganiello V \& Stratakis CA 2014 Clinical and molecular genetics of the phosphodiesterases (PDEs). Endocrine Reviews 35 195-233. (doi:10.1210/er.2013-1053)

Baviera AM, Zanon NM, Navegantes LC \& Kettelhut IC 2010 Involvement of cAMP/Epac/PI3K-dependent pathway in the antiproteolytic effect of epinephrine on rat skeletal muscle. Molecular and Cellular Endocrinology 315 104-112. (doi:10.1016/j.mce.2009.09.028)

Bender AT \& Beavo JA 2006 Cyclic nucleotide phosphodiesterases: molecular regulation to clinical use. Pharmacological Reviews $\mathbf{5 8}$ 488-520. (doi:10.1124/pr.58.3.5)

Bian H, Zhang J, Wu P, Varty LA, Jia Y, Mayhood T, Hey JA \& Wang P 2004 Differential type 4 cAMP-specific phosphodiesterase (PDE4) expression and functional sensitivity to PDE4 inhibitors among rats, monkeys and humans. Biochemical Pharmacology 68 2229-2236. (doi:10.1016/ j.bcp.2004.08.014)

Bickar D \& Reid PD 1992 A high-affinity protein stain for western blots, tissue prints, and electrophoretic gels. Analytical Biochemistry 203 109-115. (doi:10.1016/0003-2697(92)90049-D)

Brito IR, Lima IM, Saraiva MV, Silva CM, Magalhaes-Padilha DM, Araujo VR, Barreto Luz V, Barbalho Silva AW, Campello CC, Silva JR et al. 2012 Expression levels of mRNA-encoding PDGF receptors in goat ovaries and the influence of PDGF on the in vitro development of caprine pre-antral follicles. Reproduction in Domestic Animals 47 695-703. (doi:10.1111/j.1439-0531.2011.01946.x)

Budnik LT, Brunswig B \& Mukhopadhyay AK 1987 Atrial natriuretic factor stimulates luteal guanylate cyclase. Regulatory Peptides 19 23-34. (doi:10.1016/0167-0115(87)90071-1)

Cardona A, Saalfeld S, Schindelin J, Arganda-Carreras I, Preibisch S, Longair M, Tomancak P, Hartenstein V \& Douglas RJ 2012 TrakEM2 software for neural circuit reconstruction. PLOS ONE 7 e38011. (doi:10.1371/journal.pone.0038011)

Chen X \& Ruiz-Echevarria MJ 2013 TMEFF2 modulates the AKT and ERK signaling pathways. International Journal of Biochemistry and Molecular Biology 4 83-94.

Chen YJ, Hsiao PW, Lee MT, Mason JI, Ke FC \& Hwang J 2007 Interplay of $\mathrm{PI} 3 \mathrm{~K}$ and cAMP/PKA signaling, and rapamycin-hypersensitivity in TGF $\beta 1$ enhancement of FSH-stimulated steroidogenesis in rat ovarian granulosa cells. Journal of Endocrinology 192 405-419. (doi:10.1677/JOE-060076) 
Conti M 2002 Specificity of the cyclic adenosine $3^{\prime}, 5^{\prime}$-monophosphate signal in granulosa cell function. Biology of Reproduction 67 1653-1661. (doi:10.1095/biolreprod.102.004952)

Conti M, Hsieh M, Zamah AM \& Oh JS 2012 Novel signaling mechanisms in the ovary during oocyte maturation and ovulation. Molecular and Cellular Endocrinology 356 65-73. (doi:10.1016/j.mce. 2011.11.002)

Conti M, Mika D \& Richter W 2014 Cyclic AMP compartments and signaling specificity: role of cyclic nucleotide phosphodiesterases. Journal of General Physiology 143 29-38. (doi:10.1085/jgp.2013 11083)

Cui X, Yu S, Tamhane A, Causey ZL, Steg A, Danila MI, Reynolds RJ, Wang J, Wanzeck KC, Tang Q et al. 2015 Simple regression for correcting $\Delta C$ t bias in RT-qPCR low-density array data normalization. BMC Genomics 16 82. (doi:10.1186/s12864-015-1274-1)

De Miguel MP, Cheng L, Holland EC, Federspiel MJ \& Donovan PJ 2002 Dissection of the c-Kit signaling pathway in mouse primordial germ cells by retroviral-mediated gene transfer. PNAS 99 10458-10463. (doi:10. 1073/pnas.122249399)

Ding T, Luo A, Yang S, Lai Z, Wang Y, Shen W, Jiang J, Lu Y, Ma D \& Wang S 2012 Effects of basal media and supplements on diethylstilbestrol-treated immature mouse primary granulosa cell growth and regulation of steroidogenesis in vitro. Reproduction in Domestic Animals 47 355-364. (doi:10.1111/j.1439-0531.2011.01879.x)

Felding J, Sorensen MD, Poulsen TD, Larsen J, Andersson C, Refer P, Engell K, Ladefoged LG, Thormann T, Vinggaard AM et al. 2014 Discovery and early clinical development of 2-\{6-[2-(3,5-dichloro-4pyridyl)acetyl]-2,3-dimethoxyphenoxy\}-N-propylacetamide (LEO 29102), a soft-drug inhibitor of phosphodiesterase 4 for topical treatment of atopic dermatitis. Journal of Medicinal Chemistry 57 5893-5903. (doi:10.1021/jm500378a)

Gavalda A \& Roberts RS 2013 Phosphodiesterase-4 inhibitors: a review of current developments. Expert Opinion on Therapeutic Patents 23 997-1016. (doi:10.1517/13543776.2013.794789)

Grimaldi P, Capolunghi F, Geremia R \& Rossi P 2003 Cyclic adenosine monophosphate (CAMP) stimulation of the kit ligand promoter in sertoli cells requires an Sp1-binding region, a canonical TATA box, and a cAMPinduced factor binding to an immediately downstream GC-rich element. Biology of Reproduction 69 1979-1988. (doi:10.1095/biolreprod.103. 019471)

Hornick JE, Duncan FE, Shea LD \& Woodruff TK 2012 Isolated primate primordial follicles require a rigid physical environment to survive and grow in vitro. Human Reproduction 27 1801-1810. (doi:10.1093/ humrep/der468)

Johnson MC, Diaz HA, Stocco C, Palomino A, Devoto L \& Vega M 1999 Antisteroidogenic action of nitric oxide on human corpus luteum in vitro: mechanism of action. Endocrine 11 31-36. (doi:10.1385/ENDO: 11:1:31)

Johnson WB, Katugampola S, Able S, Napier C \& Harding SE 2012 Profiling of CAMP and CGMP phosphodiesterases in isolated ventricular cardiomyocytes from human hearts: comparison with rat and guinea pig. Life Sciences 90 328-336. (doi:10.1016/j.Ifs.2011.11.016)

Kwak HJ, Park KM, Choi HE, Chung KS, Lim HJ \& Park HY 2008 PDE4 inhibitor, roflumilast protects cardiomyocytes against NO-induced apoptosis via activation of PKA and Epac dual pathways. Cellular Signalling 20 803-814. (doi:10.1016/j.cellsig.2007.12.011)

Labeur M, Wölfel B, Panhuysen M, Stalla J, Stalla GK \& Paez-Pereda M 2010 TMEFF2: a new endogenous modulator of the CRH signaling in corticotroph cells. Experimental and Clinical Endocrinology \& Diabetes 118 13. (doi:10.1055/s-0030-1267015)

Li J, Kawamura K, Cheng Y, Liu S, Klein C, Liu S, Duan EK \& Hsueh AJ 2010 Activation of dormant ovarian follicles to generate mature eggs. PNAS 107 10280-10284. (doi:10.1073/pnas.1001198107)

Lin K, Taylor JR Jr, Wu TD, Gutierrez J, Elliott JM, Vernes JM, Koeppen H, Phillips HS, de Sauvage FJ \& Meng YG 2011 TMEFF2 is a PDGF-AA binding protein with methylation-associated gene silencing in multiple cancer types including glioma. PLoS ONE 6 e18608. (doi:10.1371/ journal.pone.0018608)

Lintern-Moore S, Peters H, Moore GP \& Faber M 1974 Follicular development in the infant human ovary. Journal of Reproduction and Fertility 39 53-64. (doi:10.1530/jrf.0.0390053)
Losco PE, Poulet FM, Kaminska-McNamara GZ \& Klein MF 2010 Myocardial and reproductive system toxicity of $\mathrm{SCH} 351591$, a selective phosphodiesterase-4 inhibitor, in CD-1 mice. Toxicologic Pathology 38 568-582. (doi:10.1177/0192623310370463)

Markholt S, Grondahl ML, Ernst EH, Andersen CY, Ernst E \& LykkeHartmann K 2012 Global gene analysis of oocytes from early stages in human folliculogenesis shows high expression of novel genes in reproduction. Molecular Human Reproduction 18 96-110. (doi:10.1093/molehr/gar083)

McKenna SD, Pietropaolo M, Tos EG, Clark A, Fischer D, Kagan D, Bao B, Chedrese PJ \& Palmer S 2005 Pharmacological inhibition of phosphodiesterase 4 triggers ovulation in follicle-stimulating hormone-primed rats. Endocrinology 146 208-214. (doi:10.1210/en.2004-0562)

Menon KM \& Menon B 2012 Structure, function and regulation of gonadotropin receptors - a perspective. Molecular and Cellular Endocrinology 356 88-97. (doi:10.1016/j.mce.2012.01.021)

Naryzhny SN 2009 Blue Dry Western: simple, economic, informative, and fast way of immunodetection. Analytical Biochemistry 392 90-95. (doi:10.1016/j.ab.2009.05.037)

Oktem O \& Urman B 2010 Understanding follicle growth in vivo. Human Reproduction 25 2944-2954. (doi:10.1093/humrep/deq275)

Park JY, Richard F, Chun SY, Park JH, Law E, Horner K, Jin SL \& Conti M 2003 Phosphodiesterase regulation is critical for the differentiation and pattern of gene expression in granulosa cells of the ovarian follicle. Molecular Endocrinology 17 1117-1130. (doi:10.1210/me. 2002-0435)

Petersen TS \& Andersen CY 2014 Simultaneous isolation of mRNA and native protein from minute samples of cells. BioTechniques $\mathbf{5 6} 229-237$.

Petersen TS, Kristensen SG, Jeppesen JV, Grondahl ML, Wissing ML, Macklon KT \& Andersen CY 2015 Distribution and function of $3^{\prime}, 5^{\prime}$-Cyclic-AMP phosphodiesterases in the human ovary. Molecular and Cellular Endocrinology 403 10-20. (doi:10.1016/j.mce.2015.01. 004)

Reinhardt RR, Chin E, Zhou J, Taira M, Murata T, Manganiello VC \& Bondy CA 1995 Distinctive anatomical patterns of gene expression for cGMP-inhibited cyclic nucleotide phosphodiesterases. Journal of Clinical Investigation 95 1528-1538. (doi:10.1172/JCl117825)

Sasseville M, Albuz FK, Cote N, Guillemette C, Gilchrist RB \& Richard FJ 2009 Characterization of novel phosphodiesterases in the bovine ovarian follicle. Biology of Reproduction 81 415-425. (doi:10.1095/biolreprod. 108.074450)

Schmittgen TD \& Livak KJ 2008 Analyzing real-time PCR data by the comparative C(T) method. Nature Protocols 3 1101-1108. (doi:10.1038/ nprot.2008.73)

Schwanhausser B, Busse D, Li N, Dittmar G, Schuchhardt J, Wolf J, Chen W \& Selbach M 2011 Global quantification of mammalian gene expression control. Nature 473 337-342. (doi:10.1038/nature10098)

Shaw TJ, Keszthelyi EJ, Tonary AM, Cada M \& Vanderhyden BC 2002 Cyclic AMP in ovarian cancer cells both inhibits proliferation and increases c-KIT expression. Experimental Cell Research 273 95-106. (doi:10.1006/ excr.2001.5426)

Shimizu-Albergine M, Tsai LC, Patrucco E \& Beavo JA 2012 cAMP-specific phosphodiesterases $8 \mathrm{~A}$ and $8 \mathrm{~B}$, essential regulators of Leydig cell steroidogenesis. Molecular Pharmacology 81 556-566. (doi:10.1124/ mol.111.076125)

Sleer LS \& Taylor CC 2007 Cell-type localization of platelet-derived growth factors and receptors in the postnatal rat ovary and follicle. Biology of Reproduction 76 379-390. (doi:10.1095/biolreprod.105.046854)

Smith PG, Wang F, Wilkinson KN, Savage KJ, Klein U, Neuberg DS, Bollag G, Shipp MA \& Aguiar RC 2005 The phosphodiesterase PDE4B limits CAMP-associated PI3K/AKT-dependent apoptosis in diffuse large B-cell lymphoma. Blood 105 308-316. (doi:10.1182/blood-2004-010240)

Tsafriri A, Chun SY, Zhang R, Hsueh AJ \& Conti M 1996 Oocyte maturation involves compartmentalization and opposing changes of cAMP levels in follicular somatic and germ cells: studies using selective phosphodiesterase inhibitors. Developmental Biology 178 393-402. (doi:10.1006/ dbio.1996.0226)

Tsai LC, Shimizu-Albergine M \& Beavo JA 2011 The high-affinity cAMPspecific phosphodiesterase $8 \mathrm{~B}$ controls steroidogenesis in the mouse adrenal gland. Molecular Pharmacology 79 639-648. (doi:10.1124/mol. 110.069104) 
Vaccari S, Weeks JL, Hsieh M, Menniti FS \& Conti M 2009 Cyclic GMP signaling is involved in the luteinizing hormone-dependent meiotic maturation of mouse oocytes. Biology of Reproduction 81 595-604. (doi:10.1095/biolreprod.109.077768)

Wang S, Yang S, Lai Z, Ding T, Shen W, Shi L, Jiang J, Ma L, Tian Y, Du X et al. 2013 Effects of culture and transplantation on follicle activation and early follicular growth in neonatal mouse ovaries. Cell and Tissue Research 354 609-621. (doi:10.1007/s00441-013-1678-7)

Warton DI \& Hui FK 2011 The arcsine is asinine: the analysis of proportions in ecology. Ecology 92 3-10. (doi:10.1890/10-0340.1)

Zaccolo M 2011 Spatial control of cAMP signalling in health and disease. Current Opinion in Pharmacology 11 649-655. (doi:10.1016/j.coph.2011.09.014)
Zhang $\mathbf{P}$, Louhio $\mathbf{H}$, Tuuri T, Sjoberg J, Hreinsson J, Telfer EE \& Hovatta $\mathbf{O}$ 2004 In vitro effect of cyclic adenosine 3', 5'-monophosphate (cAMP) on early human ovarian follicles. Journal of Assisted Reproduction and Genetics 21 301-306. (doi:10.1023/B:JARG.0000043704. 10845.87)

Received 24 August 2014

First decision 30 September 2014

Revised manuscript received 2 April 2015

Accepted 9 April 2015 